\title{
Association of facet tropism with lumbar disc herniation
}

\author{
Manish Chadha $\cdot$ Gaurav Sharma \\ Shobha S. Arora • Vivek Kochar
}

Received: 14 December 2011/Revised: 21 July 2012/ Accepted: 4 December 2012/Published online: 14 December 2012

(C) Springer-Verlag Berlin Heidelberg 2012

\begin{abstract}
Purpose Facet tropism is defined as asymmetry between left and right facet joints and is postulated as a possible cause of disc herniation. In the present study, the authors used a 3-T MRI to investigate the association between facet tropism and lumbar disc herniation at a particular motion segment. They also examined whether the disc herniated towards the side of the more coronally oriented facet joint. Methods Sixty patients (18-40 years) with single level disc herniation (L3-L4, L4-L5, or L5-S1) were included in the study. Facet angles were measured using MRI of 3-T using the method described by Karacan et al. Facet tropism was defined as difference of $10^{\circ}$ in facet joint angles between right and left sides. Normal disc adjacent to the herniated level was used as control. We also examined if disc herniated towards the side of more coronally oriented facet.

Results Twenty-five herniations were at L4-L5 level and 35 at L5-S1. Statistical analysis was performed using the Fischer Exact Test. At L4-L5 level, 6/25 cases had tropism compared to $3 / 35$ controls $(p=0.145)$. At L5-S1 level, $13 / 35$ cases had tropism as compared to $1 / 21$ controls $(p=0.0094)$. Of 19 cases having tropism, the disc had herniated towards the coronally oriented facet in six $(p=0.11)$.

Conclusion The findings of the study suggest that facet tropism is associated with lumbar disc herniation at the L5-S1 motion segment but not at the L4-L5 level.
\end{abstract}

M. Chadha · G. Sharma $(\bowtie) \cdot$ S. S. Arora · V. Kochar Department of Orthopaedics, University College of Medical Sciences and Guru Teg Bahadur Hospital, Dilshad Garden, New Delhi 110095, India

e-mail: sharmagaurav850@gmail.com
Keywords Facet tropism - Lumbar disc herniation . Facet asymmetry $\cdot$ Magnetic resonance imaging

\section{Introduction}

Asymmetry of facets (tropism), especially if it is marked, is a subject of intense interest because it has the potential to markedly alter the biomechanics of lumbar spinal movements and precipitate early degenerative changes either in the joint or adjacent intervertebral discs contributing to back pain [1]. Brailsford [2] defined facet tropism in 1928 as asymmetry between the left and right vertebral facet joint angles with one joint having a more sagittal orientation than the other does. In the past, there has been much clinical research relating tropism with degenerated apophyseal joints and low back pain [3-7].

Farfan and Sullivan [4] first suggested the correlation between facet tropism and development of lumbar disc herniation. They suggested that asymmetry of the facet joints is correlated with the development of disc herniation, because the coronally facing facet joint offers little resistance to intervertebral shear force, so the rotation occurs towards the side of the more coronally facing facet joint, and this possibly leads to additional torsional stress on the annulus fibrosus. There are numerous arguments for and against this hypothesis [8-16]. A correlation between facet asymmetry and the side of disc herniation (sagittal or coronal) is also debated $[4,12,13$, 17, 18].

Masharawi et al. [19] found asymmetry in facet orientation to be a normal characteristic in most thoracic vertebrae but not in the lumbar ones. They suggest, however, that when asymmetry in facet orientation occurs in lumbar vertebrae, it may be related to pathologic conditions [19]. 
Incidence of facet tropism in the lumbar motion segments varies from 10 to $70 \%$ [20] depending upon the criteria used for defining it. However, considering a cutoff of more than $10^{\circ}$, facet tropism has been found to be present in 14-28\% of lumbar motion segments [10, 21]. Although mean facet asymmetry increases from L3-L4 segment to the L5-S1 segment, the distribution of facet asymmetry at these levels has not been found to be significantly different $[21,22]$.

In the present study, the authors attempt to determine whether there is an association between facet tropism and the occurrence of lumbar disc herniation at that motion segment. In addition, an association between facet asymmetry and the side of disc herniation (sagittal or coronal) was also investigated.

\section{Materials and methods}

Sixty patients between the age of 18 and 40 years with single level lumbar disc herniation (L3-L4, L4-L5, or L5-S1) were included in the study. These patients underwent either conservative or operative treatment for their radiculopathy. Exclusion criteria included patients with multiple or recurrent disc herniation, associated spondylolisthesis, spina bifida, transitional vertebra, scoliosis, previous surgery, previous trauma/spinal infection, significant facetal arthropathy.

All patients presenting with typical symptoms suggestive of lumbar disc herniation such as low backache and sciatica were subjected to magnetic resonance imaging (MRI) as in the normal course of treatment (conservative or operative). An informed consent was taken from the patients for using the MRI data for the study. MRI was performed with a 3-T [(Signa HDxt) GE Healthcare] in all patients.

The slice thickness used was $4 \mathrm{~mm}$. The axial images were taken parallel to the end plate. A herniated disc was considered an extrusion (mainly focal) of disc material beyond the osseous confines of the vertebral body, resulting in the displacement of epidural fat, nerve root, or thecal sac.

MRI axial T1-weighted image on which left and right facet joints was best visualized were obtained on X-ray film with at least $80 \%$ magnification and reference lines were directly drawn on X-ray films. Angular measurements were made to the nearest half a degree. The measurements were made manually using a goniometer. Facet line was drawn between the anteromedial and posterolateral edges of the superior articular facets bilaterally. Midsagittal line was drawn passing through the centre of the disc and the centre of the base of the spinous process, dividing the vertebral body into two equal halves $(\mathrm{AO}=\mathrm{OB})$. Facet angle was measured for each side as the angle between facet line and mid sagittal line (Fig. 1).
Two orthopaedic surgeons measured the facet joint angles independently. Mean was taken as the true facet angle to minimize observer bias. Difference between right and left facet angle was recorded. Facet tropism was defined as the difference between the angle of the right and left facet more than $10^{\circ}$.

Normal disc adjacent to the herniated level was used as control (i.e. for L3-L4 disc herniation L4-L5 served as control, for L4-L5 disc herniation L5-S1 or L3-L4 served as control, for L5-S1 disc herniation L4-L5 served as control).

\section{Statistical analysis}

Observations were recorded in a standard Performa and a master chart was made of all the observations. Fischer Exact Test was utilized to determine the statistical significance of categorical variables; $p<0.05$ was considered significant. The statistical analysis for whether the disc

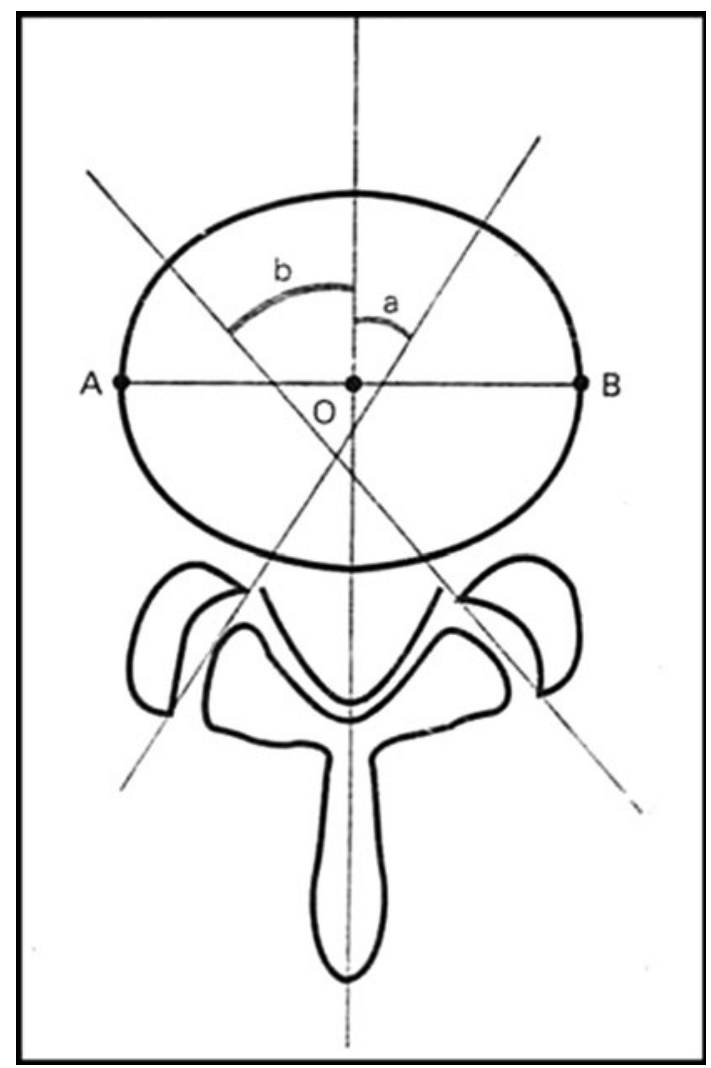

Fig. 1 Diagram of the measurement of facet tropism. A line is drawn between the two margins of each of the superior articular facets. The midsagittal line will pass through the centre of the disc $(O)$ and the centre of the base of the spinous process. The angle between the facet line and midsagittal line was measured for each side of the spine. The difference of the right $(a)$ and left $(b)$ facet angles for each patient were calculated $(\mathrm{AO}=\mathrm{OB})$ 
herniated more towards the coronal or sagittal side was performed using the test for one proportion.

Intra Class Correlation Coefficient was employed to determine the reliability/agreement between the two observers for the measurement of facet angle. A power analysis using SD of tropism measurements was performed using the $t$ test. The data were analysed using SPSS statistical Software SPSS version 16.0.

\section{Results}

Out of the total 60 cases of disc herniation (all being posterolateral), 43 discs herniated towards left and 17 herniated towards right side.

The mean age of our patients was $33.82 \pm 7.87$ years (range 18-45). The intraclass correlation coefficient was found to be 0.958 which shows good agreement between the two observers for facet angle measurement.

The maximum number of HNP were at L5-S1 $(n=35)$, while no case of HNP was seen at L3-L4. The maximum number of controls were at L4-L5 $(n=35)$, followed by L5-S1 $(n=21)$.

Overall association of facet tropism in cases (HNP) and control group at all three levels

Nineteen of the 60 cases (HNP) had tropism while only four at the control level did. The overall association of facet tropism with lumbar disc herniation was found to be highly significant by Fisher exact test. $[p$ value $=0.001$, OR 6.488 (CI 2.05-20.51)] (Table 1).

Association of facet tropism in cases (HNP) and control group at individual level

When the data were analyzed at each individual level, we found that the association was not significant at L4-L5 $[p$ value $=0.145$, OR 3.368 (CI 0.753-15.061)], while it was highly significant at L5-S1 by Fishers exact test $[p$ value $=0.0094$, OR 11.818 (CI 1.415-98.674)]. Since we did not have any case at L3-4 level, statistical analysis was not possible for that level. Figures 2 and 3 illustrate cases with and without facet tropism at the level of disc herniation (Table 2).

Table 1 Overall association of facet tropism in cases (HNP) and control group at all three levels

\begin{tabular}{llll}
\hline & Tropism present & Tropism absent & $p$ value \\
\hline HNP & 19 & 41 & 0.001 \\
Controls & 4 & 56 & \\
\hline
\end{tabular}

Power analysis of the study using SD of tropism measurements yielded a power of $p=0.848$ which is practically significant [23].

Coronal or sagittal orientation of facet joint adjacent to side of disc herniation

Of those patients who had a HNP with facet tropism, it was determined whether the disc had herniated on the side of more coronally or sagittally oriented facet joint. Analysis revealed that the disc had herniated towards the sagittally oriented facet in 13 of the 19 cases who had tropism. This was found to be statistically not significant using test of one proportion.

\section{Discussion}

There are numerous studies regarding the relationship between facet joint orientation and lumbar disc herniation [4, 9, 10, 12, 13, 16, 20, 24-27]. Asymmetrical facet joints are considered to be of importance as a cause of lumbar disc herniation by some authorities $[4,9,24]$, while others report that facet tropism has no relevance $[3,12,16]$.

Farfan and Sullivan [4] first suggested the correlation between facet tropism and development of lumbar disc herniation. Since then, various studies have yielded conflicting results concerning the association of facet tropism and intervertebral disc disease.

In 1980, Cyron and Hutton [24] postulated that tropism could lead to instability with joints rotating toward the side of the most oblique facet. Van Schaik et al. [25] were the first to use CT scans to address this issue. They measured facet asymmetry in 100 patients with backache or sciatica or both. In cases where there was less than $11^{\circ}$ of asymmetry, there was an equal distribution of herniation to the side of both the more coronally oriented and more sagittally oriented facet joint. With greater degrees of asymmetry, there was a greater incidence of unilateral disc protrusion towards the side of the more coronally oriented joint. Noren et al. [9] also concluded that facet joint asymmetry is a risk factor for the development of disc degeneration and herniation at all lumbar levels. More recently, Karacan et al. [20] in their study concluded that patient with lumbar disc herniations had the asymmetry of facet joints and that these alterations were more evident in taller patients.

In contrast, other studies have suggested that facet tropism has no clinical relevance. In 1981, Adams and Hutton [11] performed a biomechanical analysis and concluded that axial torsion was not important in the development of lumbar disc herniation. Ahmed et al. [28] showed that facet orientation has no effect on axial torque-rotation response 


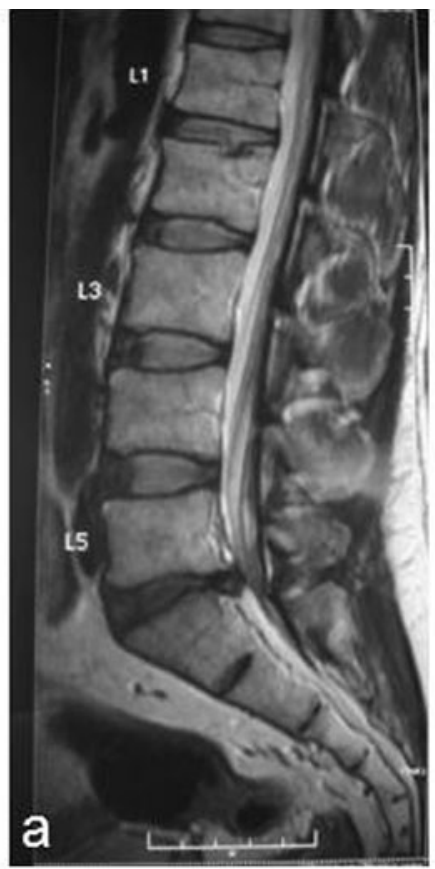

L5-S1 HNP

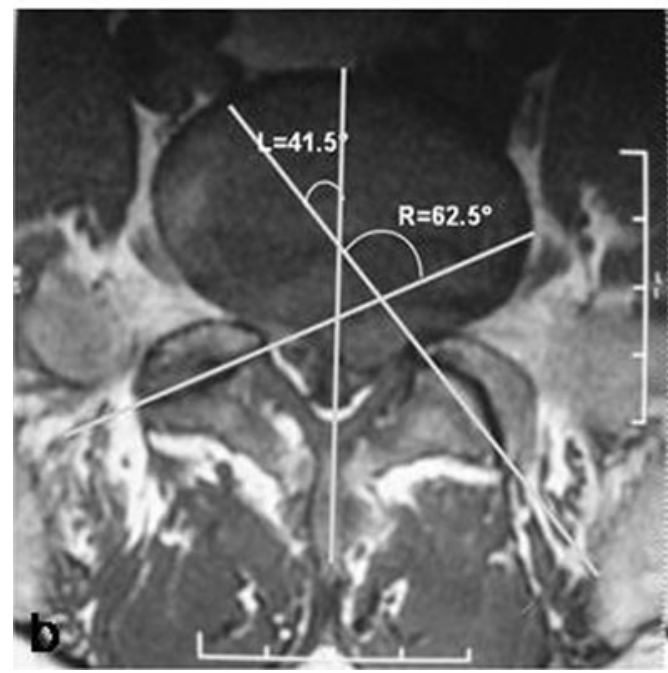

Fig. 2 a 39-year-old patient had a left sided disc herniation at L5-S1. The L4-L5 disc served as control. b The axial image shows the presence of facet tropism at the level of disc herniation, with the disc

in the lumbar spine. Regardless of their orientation, the facet joints act as a positive barrier to axial rotation.

Hagg and Wallner [13] and Cassidy [12] found no clear evidence that facet asymmetry is strongly associated with lumbar disc herniation. Vanharanta [16] suggested that there was no association between facet tropism and lumbar disc diseases including herniation and degeneration. Another study by Lee [27] showed no significant difference in facet tropism between the herniated and normal disc in both the adolescent and adult groups, except at the level L4-L5 level in the adults. In a cross-sectional study by

\section{L4-L5 Control}

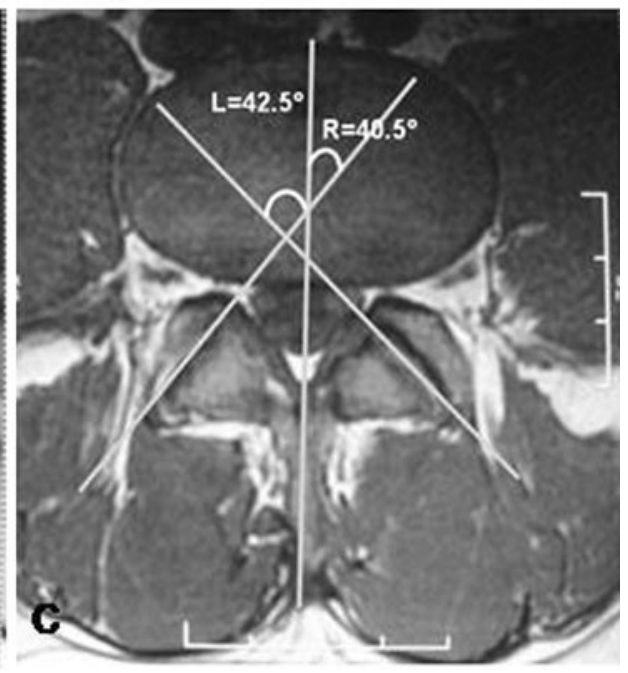

herniating towards the side of the sagittally-oriented facet. $\mathbf{c}$ The axial cut at the level of the L4-L5 disc (control) shows that the facets are symmetrical

Kunakornsawat [26] using MRI as the modality to measure the association, no statistically significant correlation was found between facet tropism and lumbar disc herniation.

The current study showed an overall statistically significant association of facet tropism with lumbar disc herniation. However, analyzing each level separately, an association of facet tropism with lumbar disc herniation was found to be statistically significant at the L5-S1 level but not at the L4-L5 level.

Anteroposterior (AP) shear of the intervertebral joints typically occurs as a motion coupled with flexion- 


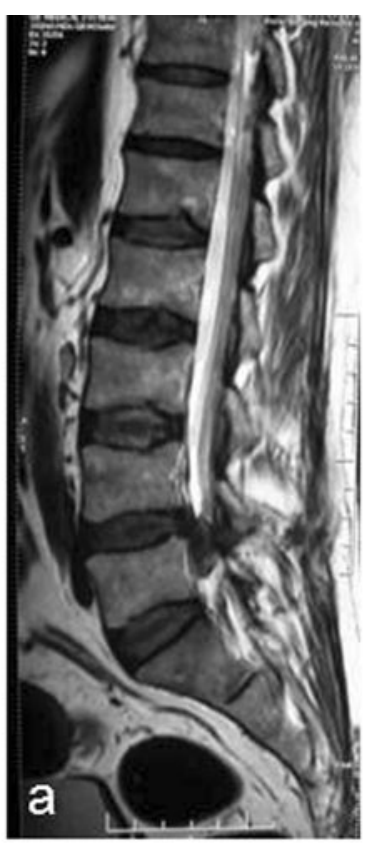

\section{L4L5 (HNP)}

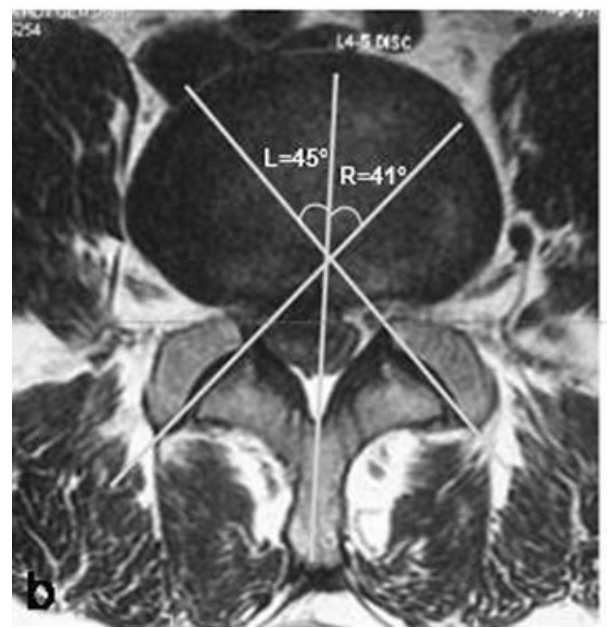

L5S1 (Control)

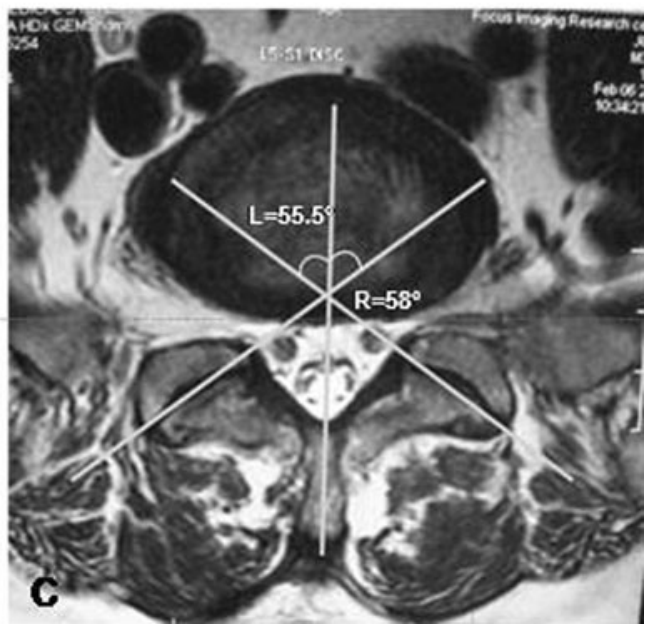

Fig. 3 a 35-year-old male patient had a right sided herniated disc at L4-L5, the L5-S1 level served as control. Axial cuts b, $\mathbf{c}$ show symmetry at both the control and HNP levels

Table 2 Association of facet tropism in cases (HNP) and control group at individual level (L3-L4, L4-L5, L5-S1)

\begin{tabular}{llllll}
\hline Level & HNP & & \multicolumn{2}{c}{ Tropism } & $p$ value \\
\cline { 3 - 5 } & & & Present & Absent & \\
\hline L3-L4 & Yes & 0 & 0 & 0 & - \\
& No & 4 & 0 & 4 & \\
L4-L5 & Yes & 25 & 6 & 19 & 0.145 \\
& No & 35 & 3 & 32 & \\
L5-S1 & Yes & 35 & 13 & 22 & 0.0094 \\
& No & 21 & 1 & 20 &
\end{tabular}

extension, such that flexion from an upright stance causes an anterior shearing motion of the superior vertebra (olisthesis), and extension causes a posterior shearing motion of the anterior vertebra (retrolisthesis) [29].

During sagittal movements of the spine (i.e., flexion/ extension), the articular facets of the zygapophyseal joints glide (i.e., translatory motion), as it is the case in any synovial joint [30]. Masharawi et al. [30] have explained as to how tropism in facet dimensions (length as well as inclination) may convert this uniplanar movement into a complex multiplanar movement. 
For example, during sagittal spinal movement (flexionextension), the more coronally orientated (C) facet joint will restrict the movement of the joint in the anteroposterior plane (Fig. 4a) leading to automatic rotation along the more sagittally oriented facet (S) (Fig. 4b).

Therefore, facet asymmetry could lead to a combined movement in the spine whenever the facet joint moves. This might lead to automatic rotation during spinal movement [30].

Whilst torsion alone may not pose a threat to the structural integrity of lumbar intervertebral discs, it appears to have a marked effect when applied in conjunction with flexion [31].

The combined action of flexion and torsion has been found to shift the nucleus posterolaterally in an asymmetric fashion, such that during flexion plus left axial rotation the nucleus is pushed against the right posterolateral annulus [32]. Using finite element analysis, Schmidt et al. [33] found that under this same loading combination, flexion plus left axial rotation, the fibres of the inner right posterolateral annulus experience the largest shear strain. Various biomechanical studies have demonstrated that the combination of flexion and torsion is capable of producing injury to the posteriolateral annulus [31, 34].

The L4-L5 interface is subjected to very little anteroposterior shearing force as compared to the L5-S1 level. There are two reasons for this. The increased inclination at the L5-S1 level leads to a larger component of anteroposterior shear force [35, 36]. (Fig. 5).

The other reason is that the beneficial role of lumbar extensor muscles in reducing the anterior shear force at L4-L5 does not hold true for the L5-S1 level, where the muscle forces generate shear forces in the anterior direction adding to those due to external load/gravity [36-38].
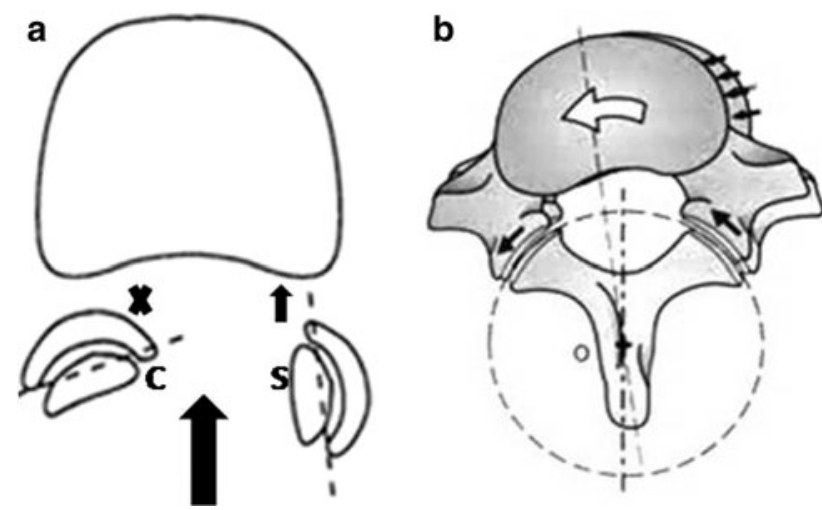

Fig. 4 During sagittal spinal movement (flexion-extension) the more coronally orientated $(\mathbf{C})$ facet joint will restrict the movement of the joint in the anteroposterior plane (a) leading to automatic rotation (b) Thus tropism could lead to a combined movement in the spine whenever the articular facet glides on each other, ending with automatic rotation during spinal movement

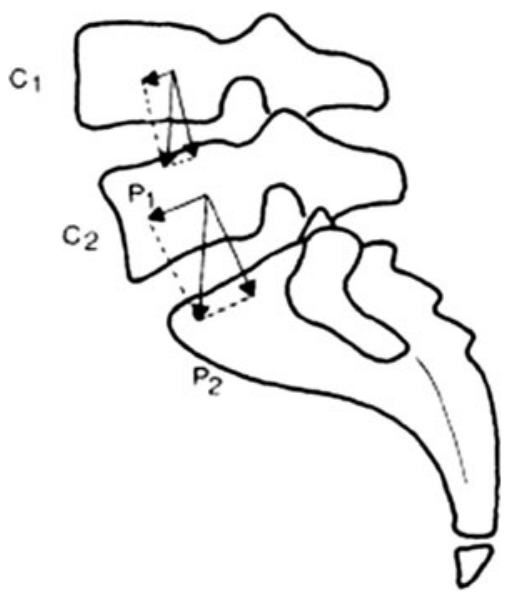

Fig. 5 The weight of the body ( $P 1$ or $P 2)$ can be broken down into component of compression perpendicular to the intervertebral disc and a component of shearing ( $C 1$ or $C 2$ ). The shearing force is small at the L4-L5 level and much greater at L5-S1

Thus, facet asymmetry at a level, which is acted upon by a larger anteroposterior shearing force, could lead to more torsion at that motion segment, thereby causing injury to the annulus fibrosus and therefore disc herniation.

We used the normal disc adjacent to the herniated disc as control. Various authors have done the same in the literature $[9,12,20,27]$. In cases of L4-L5 herniations, the adjacent (nonherniated) L5-S1 levels were scanned and compared with the same level in the patients with L5-S1 herniations. This process was reversed for cases with L5-S1 herniations. Thus, the 35 L5-S1 disc herniations have been compared to the 21 L5-S1 normal discs. Since the L5-S1 level has similar forces acting on them, we believe it is justified in comparing L5-S1 disc herniations with L5-S1 controls and similarly for the L4-L5 level.

Although facet tropism means asymmetry of the right and left facet joint, a motion segment angle difference has been variously defined. Noren et al. [9] defined facet asymmetry as a bilateral angle difference greater than $5^{\circ}$. In one biomechanical study [24], facet asymmetry was defined as a difference in facet angles greater than $1^{\circ}$. We defined facet tropism as the bilateral angle difference greater than $10^{\circ}$. Other authors have also used a cutoff of $10^{\circ}$ for defining moderate/severe face tropism $[10,21]$. We believe that $10^{\circ}$ is the ideal number for defining tropism as a smaller cutoff, e.g. $1^{\circ}$ or $4^{\circ}$ could introduce a measurement bias (whether there is actually tropism or just measurement error) as well lead to inclusion of a much larger number of motion segments, while a larger cutoff, e.g. $15-20^{\circ}$, would be present at very few levels.

In clinical studies, Farfan and Sullivan [4] and Loback [17] found a high association between the side of disc herniation and the coronally facing facet joint. 
On the other hand, Hagg and Wallner [13] and Cassidy [12] found no difference in the distribution of the more coronally or sagittally facing facet joints with respect to the side of lateral herniation. Kénési and Lesur [18] showed a correlation between facet asymmetry and the side of disc protrusion in the L5-S1 segment, which however was not present in the L4-L5 segment. They had found in their study that herniation is on the side of the more sagittal facet joint interface in three quarters of cases.

We did not find any significant difference in the distribution of the more coronally or sagittally facing facet joint with respect to the side of disc herniation. However, the number of cases where there was a significant tropism associated with a herniated disc at the same level was only 19 in our study. Though the trend in our study suggests an increased incidence of herniation towards the sagittallyoriented facet (13 out of 19), it was not statistically significant. This may be due to a smaller number of cases with asymmetry more than ten degrees. We feel that it is difficult to categorically say that there is an increased occurrence of disc herniation on the side of the more coronally or sagittally oriented facet due to the small number of such cases in our study.

The increasing obliquity of the facet joints towards a coronal orientation at more caudal levels as we have found is consistent with other published reports and clinical experience. The consistency of our measurements (good agreement between the two observers; ICC 0.958) is probably because we used 3-T MRI which improves visualization of anatomic structures (including facet joints) over 1.5-T MRI [39].

\section{Shortcomings of the study}

The number of cases where there was positive tropism at the level with the herniated disc $(n=19)$ was small, hence it is not possible to categorically say if there is an association between the side of disc herniation (coronal or sagittal) or not.

We also used the normal disc adjacent to the herniated level as the control group. It would be best if the controls were obtained by performing MRI for asymptomatic individuals.

\section{Conflict of interest None.}

\section{References}

1. Tulsi RS, Hermanis GM (1993) A study of the angle of inclination and facet curvature of superior lumbar zygapophyseal facets. Spine 18:1311-1317

2. Brailsford JF (1928) Deformities of the lumbosacral region of the spine. Br J Surg 16:562-627
3. Badgley CE (1941) The articular facets in relation to low-back pain and sciatic radiation. J Bone Jt Surg 23:481-496

4. Farfan HF, Sullivan JD (1967) The relation of facet orientation to intervertebral disc failure. Can J Surg 10:179-185

5. Ferguson AB (1934) The clinical and roentgenographic interpretation of lumbosacral anomalies. Radiology 22:548-558

6. Howard LG (1942) Low back pain and the lumbosacral joint. Med Clin N Am 26:1551-1579

7. Hirsch C (1966) Etiology and pathogenesis of low back pain. Isr J Med Sci 2:362-370

8. Park JB, Chang H, Kim KW, Park SJ (2001) Facet tropism: a comparison between far lateral and posterolateral lumbar disc herniations. Spine 26:677-679

9. Noren R, Trafimow J, Andersson GB, Huckman MS (1991) The role of facet joint tropism and facet angle in disc degeneration. Spine 16:530-532

10. Boden SD, Riew KD, Yamaguchi K, Branch TP, Schellinger D, Wiesel SW (1996) Orientation of the lumbar facet joints: association with degenerative disc disease. J Bone Jt Surg Am 78: 403-411

11. Adams MA, Hutton WC (1981) The relevance of torsion to the mechanical derangement of the lumbar spine. Spine 6:241-248

12. Cassidy JD, Loback D, Yong-Hing K, Tchang S (1992) Lumbar facet joint asymmetry. Intervertebral disc herniation. Spine 17: $570-574$

13. Hagg O, Wallner A (1990) Facet joint asymmetry and protrusion of intervertebral disc. Spine 15:356-359

14. Ishihara H, Matsui H, Osada R, Ohshima H, Tsuji H (1997) Facet joint asymmetry as a radiologic feature of lumbar intervertebral disc herniation in children and adolescents. Spine 22:2001-2004

15. Ko HY, Park BK (1997) Facet tropism in lumbar motion segments and its significance in disc herniation. Arch Phys Med Rehabil 78:1211-1214

16. Vanharanta H, Floyd T, Ohnmeiss D (1993) The relationship of facet tropism to degenerative disc disease. Spine 18:1000-1005

17. Loback D, Young-Hing K, Cassidy D, Tchang S (1985) The relationship between facet orientation and lumbar disc herniation: the role of torsion in intervertebral disc failure. Orthop Trans 9:560-563

18. Kénési C, Lesur E (1985) Orientation of the articular processes at L4, L5, and S1. Possible role in pathology of the intervertebral disc. Anat Clin 7:43-47

19. Masharawi Y, Rothschild B, Dar G et al (2004) Facet orientation in the thoraco lumbar spine: three-dimensional anatomic and biomechanical analysis. Spine 29:1755-1763

20. Karacan I, Aydin T, Sahin Z, Cidem M, Koyuncu H, Aktas I, Uludaq M (2004) Facet angles in lumbar disc herniation: their relation to anthropometric features. Spine 29:1132-1136

21. Do DH, Taghavi CE, Fong W, Kong MH, Morishita Y, Wang JC (2011) The relationship between degree of facet tropism and amount of dynamic disc bulge in lumbar spine of patients symptomatic for low back pain. Eur Spine J 20:71-78

22. Kong MH, He W, Tsai YD, Chen NF, Keorochana G, Do DH, Wang JC (2009) Relationship of facet tropism with degeneration and stability of functional spine unit. Yonsei Med J 50:624-629

23. Lau CC, Kuk F (2011) Enough is enough: a primer on power analysis in study designs. Hear J 64:30-39

24. Cyron BM, Hutton WC (1980) Articular tropism and stability of the lumbar spine. Spine 5:168-172

25. Van Schaik JPJ, Verbiest H, van Schanik FDJ (1984) The orientation and shape of the lower lumbar facet joints: a computed tomographic study of their variation in 100 patients with low back pain and a discussion of their possible clinical implications. In: MJ Donovan Post (ed) Computed tomography of the Spine. Williams \& Wilkins, Baltimore, pp 495-505 
26. Kunakornsawat S, Ngamlamaidt K, Tungsiripat R, Prasartritha T (2007) The relationship of facet tropism to lumbar disc herniation. J Med Assoc Thai 90:1337-1341

27. Lee DY, Ahn Y, Lee SH (2006) The influence of facet tropism on herniation of the lumbar disc in adolescents and adults. J Bone Jt Surg Br 88:520-523

28. Duncan NA, Ahmed AM, Ahmed AM (1991) The role of axial rotation in the etiology of unilateral disc prolapse. An experimental and finite-element analysis. Spine 16:1089-1098

29. Lu WW, Luk KD, Holmes AD, Cheung KM, Leong JC (2005) Pure shear properties of lumbar spinal joints and the effect of tissue sectioning on load sharing. Spine 30:204-209

30. Masharawi Y, Rothschild B, Salame K, Dar G, Peleg S, Hershkovitz I (2005) Facet tropism and interfacet shape in the thoracolumbar vertebrae: characterization and biomechanical interpretation. Spine 30:281-292

31. Veres SP, Robertson PA, Broom ND (2010) The influence of torsion on disc herniation when combined with flexion. Eur Spine J 19:1468-1478

32. Fazey PJ, Song S, Monsas S, Johansson L, Haukalid T, Price RI, Singer KP (2006) An MRI investigation of intervertebral disc deformation in response to torsion. Clin Biomech (Bristol, Avon) $21: 538-542$
33. Schmidt H, Kettler A, Heuer F, Simon U, Claes L, Wilke HJ (2007) Intradiscal pressure, shear strain, and fiber strain in the intervertebral disc under combined loading. Spine 32:748-755

34. Drake JD, Aultman CD, McGill SM, Callaghan JP (2005) The influence of static axial torque in combined loading on intervertebral joint failure mechanics using a porcine model. Clin Biomech 20:1038-1045

35. Kingma I, Bosch T, Bruins L, van Dieën JH (2004) Foot positioning instruction, initial vertical load position and lifting technique: effects on low back loading. Ergonomics 47:1365-1385

36. Arjmand N, Shirazi-Adl A (2005) Biomechanics of changes in lumbar posture in static lifting. Spine 30:2637-2648

37. Kingma I, Staudenmann D, van Dieen JH (2007) Trunk muscle activation and associated lumbar spine joint shear forces under different levels of external forward force applied to the trunk. J Electromyogr Kinesiol 17:14-24

38. Potvin JR, Norman RW, McGill SM (1991) Reduction in anterior shear forces on the L4/L5 disc by the lumbar musculature. Clin Biomech 6:88-96

39. Zhao J, Krug R, Xu D, Lu Y, Link TM (2009) MRI of the spine: image quality and normal-neoplastic bone marrow contrast at $3 \mathrm{~T}$ versus 1.5 T. AJR 192:873-880 